\title{
Results from the OPERA Experiment in the CNGS Beam
}

\author{
Alessandro Paoloni ${ }^{(D)}$ on Behalf of OPERA Collaboration † \\ INFN_-Istituto Nazionale di Fisica Nucleare-Laboratori Nazionali di Frascati, I-00044 Frascati (RM), Italy; \\ alessandro.paoloni@lnf.infn.it \\ † The OPERA collaboration author list: Agafonova, N.; Alexandrov, A.; Anokhina, A.; Aoki, S.; Ariga, A.; \\ Ariga, T.; Bertolin, A.; Bozza, C.; Brugnera, R.; Buonaura, A.; Buontempo, S.; Chernyavskiy, M.; Chukanov, A.; \\ Consiglio, L.; D'Ambrosio, N.; De Lellis, G.; De Serio, M.; Del Amo Sanchez, P.; Di Crescenzo, A.; \\ Di Ferdinando, D.; Di Marco, N.; Dmitrievsky, S.; Dracos, M.; Duchesneau, D.; Dusini, S.; Dzhatdoev, T.; \\ Ebert, J.; Ereditato, A.; Fini, R. A.; Fornari, F.; Fukuda, T.; Galati, G.; Garfagnini, A.; Gentile, V.; Goldberg, J.; \\ Gorbunov, S.; Gornushkin, Y.; Grella, G.; Guler, A. M.; Gustavino, C.; Hagner, C.; Hara, T.; Hayakawa, T.; \\ Hollnagel, A.; Ishiguro, K.; Iuliano, A.; Jakovčić, K.; Jollet, C.; Kamiscioglu, C.; Kamiscioglu, M.; Kim, S. H.; \\ Kitagawa, N.; Kliček, B.; Kodama, K.; Komatsu, M.; Kose, U.; Kreslo, I.; Laudisio, F.; Lauria, A.; Ljubičić, A.; \\ Longhin, A.; Loverre, P.F.; Malgin, A.; Mandrioli, G.; Matsuo, T.; Matveev, V.; Mauri, N.; Medinaceli, E.; \\ Meregaglia, A.; Mikado, S.; Miyanishi, M.; Mizutani, F.; Monacelli, P.; Montesi, M. C.; Morishima, K.; \\ Muciaccia, M. T.; Naganawa, N.; Naka, T.; Nakamura, M.; Nakano, T.; Niwa, K.; Ogawa, S.; Okateva, N.; \\ Ozaki, K.; Paparella, L.; Park, B. D.; Pasqualini, L.; Pastore, A.; Patrizii, L.; Pessard, H.; Podgrudkov, D.; \\ Polukhina, N.; Pozzato, M.; Pupilli, F.; Roda, M.; Roganova, T.; Rokujo, H.; Rosa, G.; Ryazhskaya, O.; Sato, O.; \\ Schembri, A.; Shakiryanova, I.; Shchedrina, T.; Shibayama, E.; Shibuya, H.; Shiraishi, T.; Simone, S.; \\ Sirignano, C.; Sirri, G.; Sotnikov, A.; Spinetti, M.; Stanco, L.; Starkov, N.; Stellacci, S. M.; Stipčević, M.; \\ Strolin, P.; Takahashi, S.; Tenti, M.; Terranova, F.; Tioukov, V.; Vasina, S.; Vilain, P.; Voevodina, E.; Votano, L.; \\ Vuilleumier, J. L.; Wilquet, G.; Yoon, C. S.
}

Received: 31 October 2018; Accepted: 3 December 2018; Published: 7 December 2018

check for updates

\begin{abstract}
The OPERA experiment was designed to observe $v_{\mu} \rightarrow v_{\tau}$ oscillations through $\tau$ appearance on the CERN Neutrino to Gran Sasso (CNGS) beam over a baseline of $730 \mathrm{~km}$. OPERA was a hybrid experiment composed of lead plates and emulsion layers acting as a target for neutrino interactions. The experiment was complemented with electronic detectors: scintillator strips used as Target Trackers and muon spectrometers. A review of the OPERA final results is presented in this paper.
\end{abstract}

Keywords: neutrino oscillations; OPERA experiment; Tau neutrino cross section; cosmic ray physics

\section{Introduction}

After more than 60 years since the first direct observation of anti- $v_{e}$ from reactors, neutrinos are still the least known particles of the Standard Model, which foresees the existence of three neutrinos, one for each charged lepton. As a consequence of the fact that mass eigenstates $\left(v_{1}, v_{2}, v_{3}\right)$ are not eigenstates of the weak interactions mediated by W bosons $\left(v_{e}, v_{\mu}, v_{\tau}\right)$, neutrino flavor oscillations may happen, depending on the difference of the squared masses, $\Delta m^{2}$, and on the $3 \times 3$ mixing matrix, $U$, parametrized with three rotation angles and one $\mathrm{CP}$-violating phase. Even if the absolute mass scale has not yet been measured, the two $\Delta m^{2}$ values are well known from neutrino oscillation experiments, as well as the mixing matrix, all except for the phase. Nowadays there are many experiments that have observed neutrino oscillations, both from natural (solar and atmospheric) and artificial (nuclear reactors and neutrino beams) sources. A complete list is beyond the aim of this paper; the interested reader is referred to Reference [1].

This paper is a review of the latest results from the OPERA experiment, designed to observe, on a muon neutrino beam, oscillations in tau neutrinos induced by $\Delta m_{a t m}^{2}$ and $\theta_{23}$, the parameters 
measured for the first time by Super-Kamiokande from the observation of oscillations in atmospheric neutrinos, produced by primary cosmic ray interactions in the atmosphere [2].

The design of the experiment, including the choice of the $730 \mathrm{~km}$ baseline (the distance between CERN and Gran Sasso) and the energy of the beam (17 GeV on average), was optimized to observe $v_{\tau}$ appearance in a pure $v_{\mu}$ beam. For a baseline $\mathrm{L}=730 \mathrm{~km}$, the oscillation probability, proportional to $\sin ^{2}\left(1.27 \Delta m^{2}\left(\mathrm{eV}^{2}\right) L(\mathrm{~km}) / E(\mathrm{GeV})\right)$, is indeed maximum for neutrino energies $\mathrm{E} \sim 1 \mathrm{GeV}$, below the energy threshold for $\tau$ production in $v_{\tau}$ Charged Current (CC) interactions (around $3.5 \mathrm{GeV}$ ). In addition, since the $\tau$ identification is based on its mean free path in the laboratory frame, the selection efficiency increases with the energy.

A complete description of the beam and of the experiment can be found in Reference [3]. It is worth noticing that, thanks to the use of nuclear emulsions, OPERA is a unique experiment, able to identify the CC interactions of all the three neutrino flavors $\left(v_{e}, v_{\mu}\right.$, and $\left.v_{\tau}\right)$.

\section{Materials and Methods}

In this section a brief description of the experiment and of the emulsion scanning procedures (a real part of the data taking) is given. Other details, specific to each single analysis, are given in the respective sections.

\subsection{The OPERA Experiment}

The OPERA experiment, located in the underground Gran Sasso laboratories, took data from 2008 to 2012 on the CNGS (CERN Neutrino to Gran Sasso) beam. The neutrino interactions and the $\tau$ decay vertices were reconstructed by means of the Emulsion Cloud Chamber (ECC) technique, with $1 \mathrm{~mm}$ thick lead plates being alternated with nuclear emulsion layers, combining the high mass density of the lead with the spatial precision $(\sim \mu \mathrm{m})$ of the emulsions. One ECC brick, $12 \times 10 \mathrm{~cm}^{2}$ wide, was composed of 56 lead plates and 57 emulsion layers. The ECC bricks also provided momentum measurements through multiple Coulomb scattering, electromagnetic showers energy measurements, and particle identification. The bricks arranged into walls were alternated with Target Tracker (TT) modules made from crossed scintillator strips, with the light collected by WLS fibers and read by multi-anode photomultipliers. The experiment was divided into two supermodules, each made from 31 ECC walls alternated with TT layers and followed by a muon spectrometer in order to reduce the charm background. The muon spectrometers were composed of an iron-core dipole magnet with drift tubes used as precision trackers and 22 layers of resistive plate chambers (RPC) inside the magnetized iron. The experiment has already been decommissioned.

\subsection{Events Classification and Scanning}

For $1.8 \times 10^{20}$ protons on target collected at CERN, about 19,000 neutrino interactions were recorded by the electronic detectors, and, out of them, more than 7000 events located in the ECC bricks. Neutrino interaction events were selected in coincidence with the CNGS spill time. Electronics detector data were also used for the classification of the events (internal or external to the target, with or without muons) and for finding the bricks containing the neutrino vertex (brick finding). ECC bricks were endowed downstream with an external pair of emulsion layers, the so-called Changeable Sheets (CS), used for confirmation of the TT-based brick finding predictions. The emulsions of the bricks with confirmed predictions were developed and scanned: tracks were followed back from the CS (scan back) until a stopping point was found. Neutrino vertexes were fully reconstructed inside a scanning area of about $1 \mathrm{~cm}^{2}$ in five emulsion layers upstream and 10 downstream (volume scan) and a decay search procedure, described in Reference [4], was used to identify $\tau$ candidates. Tracks and showers were then followed inside the considered brick and, for $\tau$ candidates, in the downstream bricks (scan forth). 


\section{Results}

\section{1. $v_{\mu} \rightarrow v_{\tau}$ Oscillations}

The main OPERA target was the research of $v_{\mu} \rightarrow v_{\tau}$ oscillations, performed at the beginning by means of a $v_{\tau} \mathrm{CC}$ event selection based on stringent kinematic cuts. The full analysis chain was carefully described in Reference [5] and its results, on the full data sample, published in Reference [6], where $5.1 \sigma$ significance on $v_{\tau}$ appearance was reported, based on the observation of five candidates with an estimated background of $0.25 \pm 0.05$ events (and $2.64 \pm 0.53$ expected $v_{\tau}$ events from $v_{\mu}$ oscillations). An example of $\tau$ candidate is shown in Figure 1.

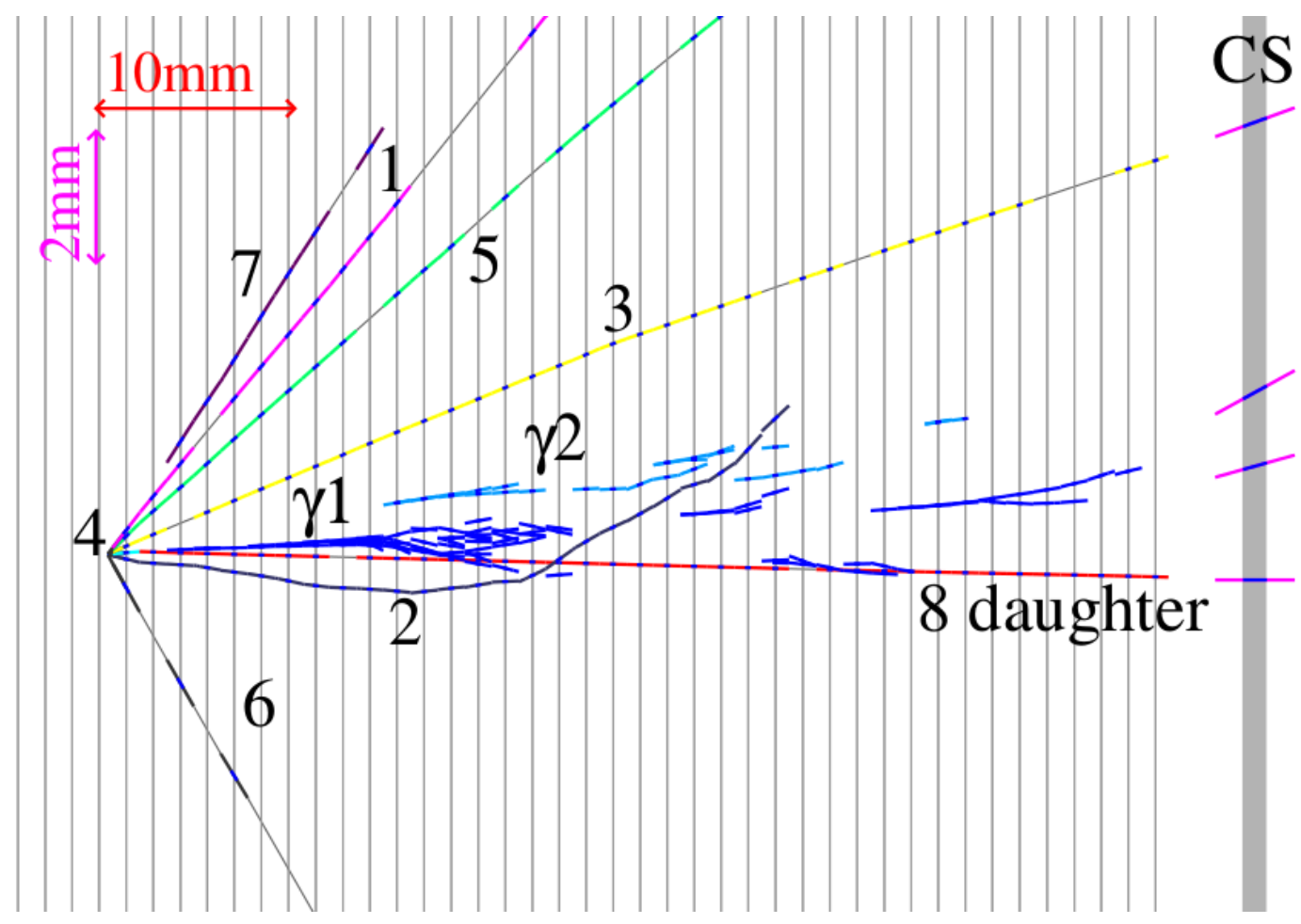

Figure 1. The first $\tau$ candidate observed by OPERA. The candidate, visible as a small cyan-colored track connected to the interaction vertex and labeled with 4 , undergoes a decay, within $1 \mathrm{~mm}$, into a charged pion, colored in red and labeled with 8 , and two $\gamma$, whose invariant mass is compatible with that of a $\pi^{0}$. The charged particles in the event, are composed by aligned track segments reconstructed in consecutive emulsion layers.

In order to increase the statistics of $\tau$ candidates, the previous selection has been replaced by a new one, based on looser cuts and reported on Table 1, resulting in ten candidates (including the previous five "golden" events) with an increased background of $2.0 \pm 0.4$ events for $6.8 \pm 1.4$ expected $\tau$. A Boosted Decision Tree (BDT) classifier was used to build a likelihood, and finally a significance of $6.1 \sigma$ on tau appearance [7] was obtained. The BDT response for the ten $\tau$ candidates is displayed in Figure 2, together with the MonteCarlo (MC) predictions for signal and background. In Figure 3, the visible energy distribution of the events is instead shown. One of the additional five selected $\tau$ is noticeably produced by a $v_{\tau} \mathrm{CC}$ interaction in association with a charmed particle. 

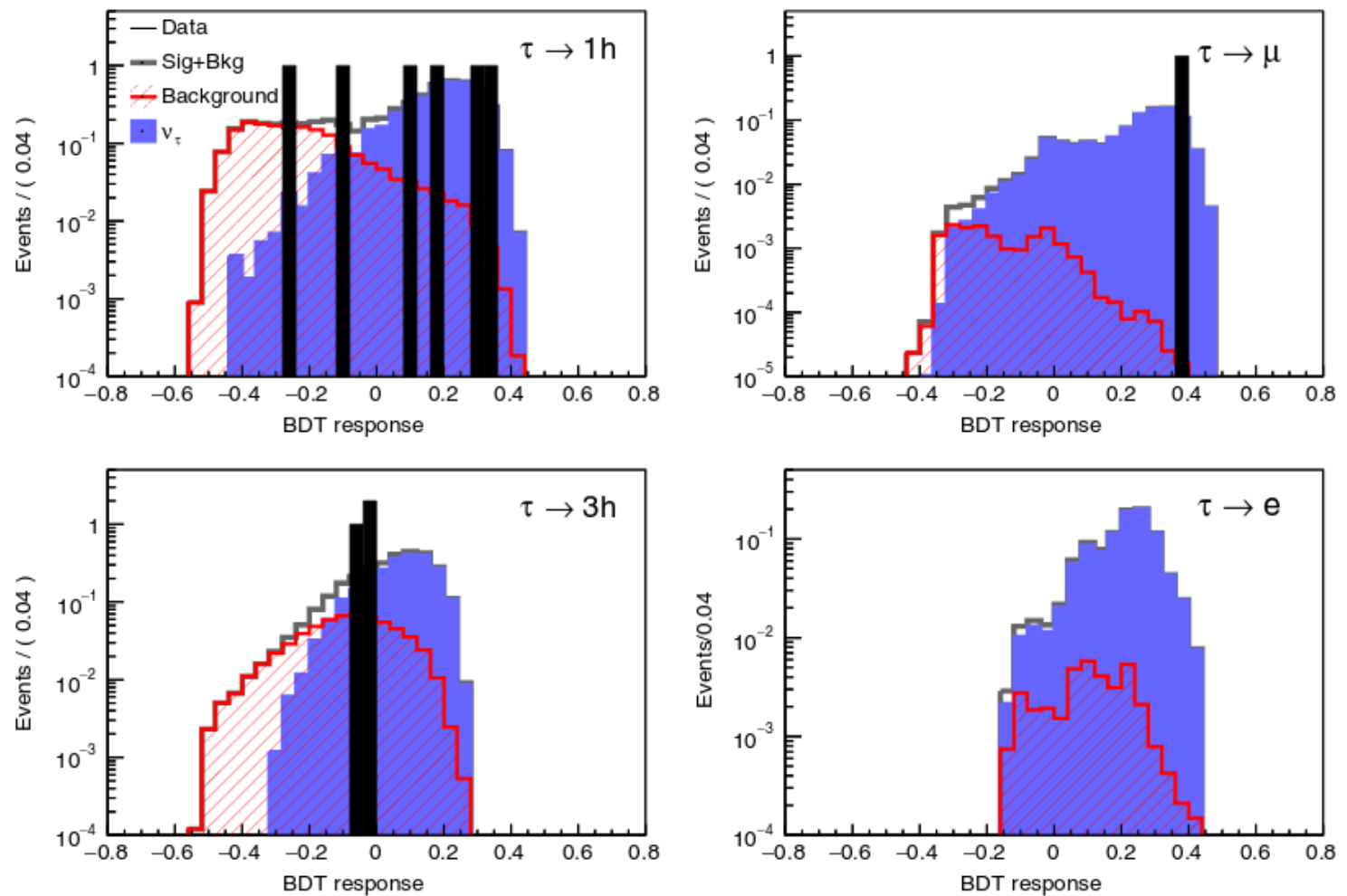

Figure 2. Boosted Decision Tree (BDT) response for the ten $\tau$ candidates. The expected distributions are shown for signal and background. All the four considered $\tau$ decay channels, muonic, electronic, single prong hadronic, and three prongs hadronic, have been considered.

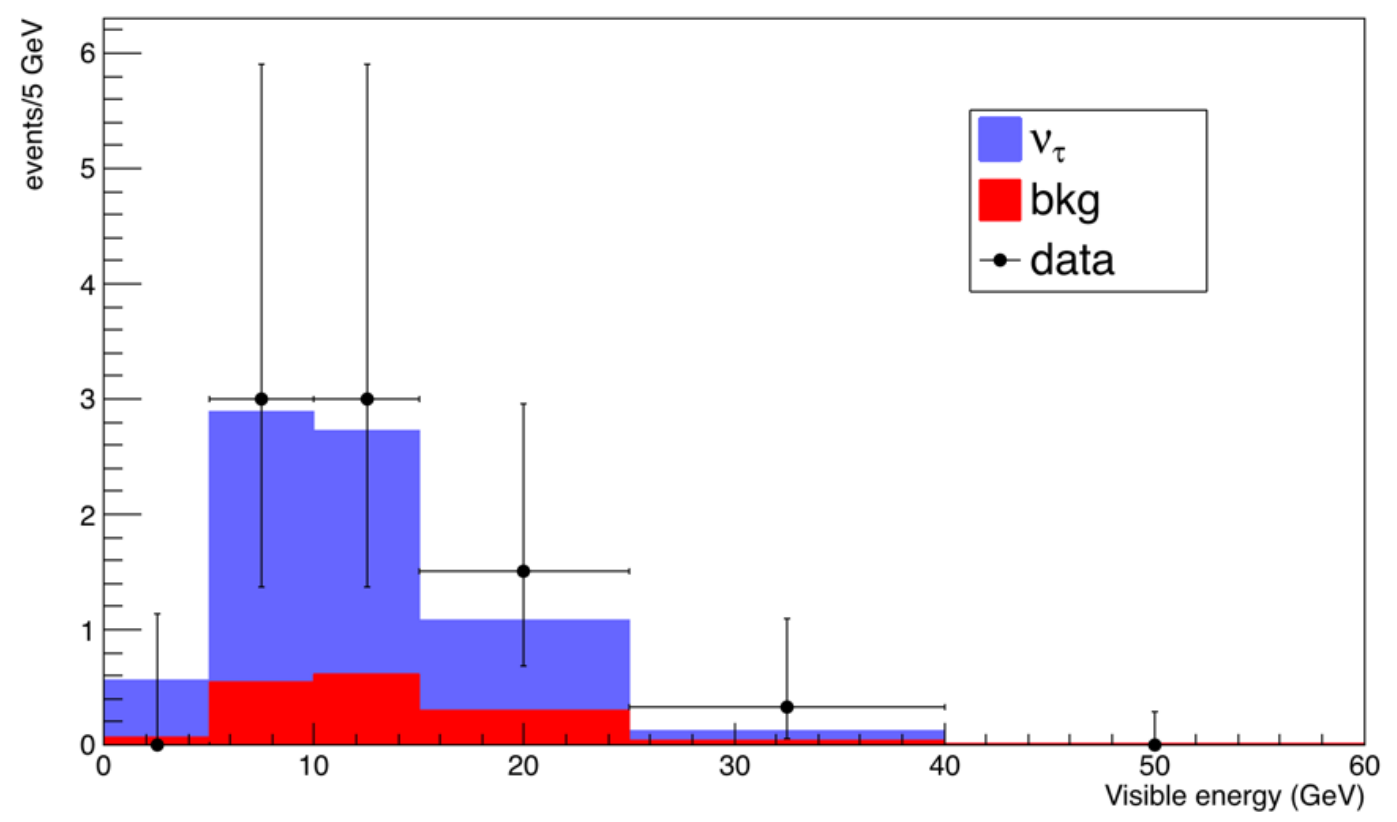

Figure 3. Visible energy distribution of the $10 \tau$ candidates. MonteCarlo predictions for signal and background are also shown. 
Table 1. Selection cuts for the different $\tau$ decay topologies. $z_{\text {dec }}$ is defined as the distance between the decay vertex and the downstream face of the lead plate containing the primary vertex. $\theta_{\text {kink }}$ is the angle between the $\tau$ candidate and its daughters. $p_{2 r y}$ is the total momentum of the visible tracks coming out from the decay vertex. For one prong decays, $p_{2 r y}^{T}$ is the daughter transverse momentum with respect to the $\tau$ candidate direction. For muonic decays, also the charge of the daughter muon, Charge $2 r y$, is considered.

\begin{tabular}{ccccc}
\hline Variable & $\boldsymbol{\tau} \rightarrow \mathbf{1} \mathbf{h}$ & $\boldsymbol{\tau} \rightarrow \mathbf{3} \mathbf{h}$ & $\boldsymbol{\tau} \rightarrow \boldsymbol{\mu}$ & $\boldsymbol{\tau} \rightarrow \boldsymbol{e}$ \\
\hline$z_{\text {dec }}(\mathrm{mm})$ & $<2.6$ & $<2.6$ & $<2.6$ & $<2.6$ \\
$\theta_{\text {kink }}(\mathrm{rad})$ & $<0.02$ & $<0.02$ & $<0.02$ & $<0.02$ \\
$p_{2 r y}(\mathrm{GeV} / \mathrm{c})$ & $>1$ & $>1$ & {$[1,15]$} & $>1$ \\
$p_{2 r y}^{T}(\mathrm{GeV} / \mathrm{c})$ & $>0.15$ & & $>0.1$ & $>0.1$ \\
Charge $_{2 r y}$ & & \multicolumn{3}{c}{ Negative } \\
& & \multicolumn{3}{c}{ or unknown } \\
\hline
\end{tabular}

The background is due mainly to charm production in $v_{\mu}$ Charged Current interactions, when the muon at the primary vertex is not identified. This background is estimated by means of MC simulations tuned on CHORUS data and validated with OPERA events containing charmed particles [4]. The charm background is also used to control the $\tau$ selection efficiency, given the similar life-times. Other background sources are hadron re-interactions in muon-less events and large angle scattering of muons in $v_{\mu}$ CC interactions. Both are estimated by MC simulations (based on Fluka and Geant4, respectively) validated on OPERA data and pion test beams, for hadron re-interactions, and on previous experimental results for large angle muon scattering [8].

Fixing the $v_{\tau}$ cross section to the one provided in the default configuration of GENIE v2.6 neutrino MonteCarlo generator [9], a value of $\left|\Delta m_{32}^{2}\right|=\left(2.7_{-0.6}^{+0.7}\right) \times 10^{-3} \mathrm{eV}^{2}$ at $68 \% \mathrm{CL}$ was estimated at a full mixing angle, compatible with the values measured with greater precision by disappearance experiments. Fixing instead the $\left|\Delta m_{32}^{2}\right|$ value to $2.5 \times 10^{-3} \mathrm{eV}^{2}$ and assuming a full mixing angle, the $v_{\tau}$ CC interaction cross section on lead was measured, averaging over the CNGS neutrino flux: $\left\langle\sigma_{v_{\tau}}\right\rangle=\left(5.1_{-2.0}^{+2.4}\right) \times 10^{-36} \mathrm{~cm}^{2}$, compatible within the errors with the default implementation in GENIE v2.6 $\left(<\sigma_{G}>=(4.29 \pm 0.04) \times 10^{-36} \mathrm{~cm}^{2}\right)$, derived assuming the Standard Model. This measurement is the first one performed on a neutrino beam with a small contamination of anti-neutrinos ( $2 \%$ in terms of interactions on target).

The observed $v_{\tau}$ events can be used to constrain theories beyond the Standard Model. Anomalies observed in LSND, MiniBoone, and reactor experiments suggest the possible existence of one or more sterile neutrinos with a mass splitting value much larger than the atmospheric one. In the following, we will refer to the simplest case, in which there is only one additional sterile neutrino. Consistency with solar and atmospheric neutrino oscillation results requires the mass of this neutrino to be separated from those of the three standard ones $(3+1$ model $)$. The mixing matrix is composed of six rotation angles and three $\mathrm{CP}$-violating phases, while the additional squared mass difference, $\Delta m_{41}^{2}$, causes oscillations at $L / E$ ratios much shorter than the OPERA one. We already published our results in the plane $\Delta m_{41}^{2}$ vs. $\sin ^{2}\left(2 \theta_{\mu} \tau\right)$ (defined as $4\left|U_{\mu 4}\right|^{2}\left|U_{\tau 4}\right|^{2}, U_{\mu 4}$ and $U_{\tau 4}$ being mixing matrix elements), based on the number of $\tau$ observed on a data sub-sample with the previous cut-based selection [10]; with the same selection, using the full data sample, an upper limit on $\sin ^{2}\left(2 \theta_{\mu} \tau\right)$ of 0.119 is derived at $90 \% \mathrm{CL}$ for $\Delta m_{41}^{2}>0.5 \mathrm{eV}^{2}$.

\section{2. $v_{\mu} \rightarrow v_{e}$ Oscillations}

Though the CNGS beam energy and baseline were optimized for $v_{\tau}$ appearance detection, the $v_{e}$ appearance was also measured by OPERA, exploiting the ECC micro-metric resolution in electro-magnetic shower reconstruction. The discrimination power between showers originated by electrons and photons also allowed an excellent rejection of the background due to Neutral Current (NC) neutrino interaction events. The OPERA final results were recently published in Reference [11]. 
In Figure 4-left, the visible energy distribution is shown for the $35 v_{e}$ CC events observed in OPERA, which are compatible with the $34.3 \pm 3.4$ expected events. The main source of the background is the $v_{e}$ contamination of the CNGS beam ( $1 \%$ in terms of neutrino interactions in the target, accounting for $30.7 \pm 3.1$ expected events). Other backgrounds are neutral current interactions with mis-identified $\pi^{0}$ or $\tau$ electronic decays amounting to a total of $1.2 \pm 0.5$ expected events. No evidence for $v_{e}$ appearance is found and an upper limit of 0.43 at $90 \%$ CL is obtained for $\sin ^{2}\left(2 \theta_{13}\right)$. An analysis based on the $3+1$ model was performed and the results are displayed in Figure 4-right in the plane $\Delta m_{41}^{2}$ vs. $\sin ^{2}\left(2 \theta_{\mu e}\right)$ (defined as $4\left|U_{\mu 4}\right|^{2}\left|U_{e 4}\right|^{2}$, where $U_{\mu 4}$ and $U_{e 4}$ are the mixing matrix elements). The eventual existence of a sterile neutrino would enhance the $v_{\mu} \rightarrow v_{e}$ oscillation probability for which we have no evidence; therefore, an exclusion region is derived for the above quoted parameters.
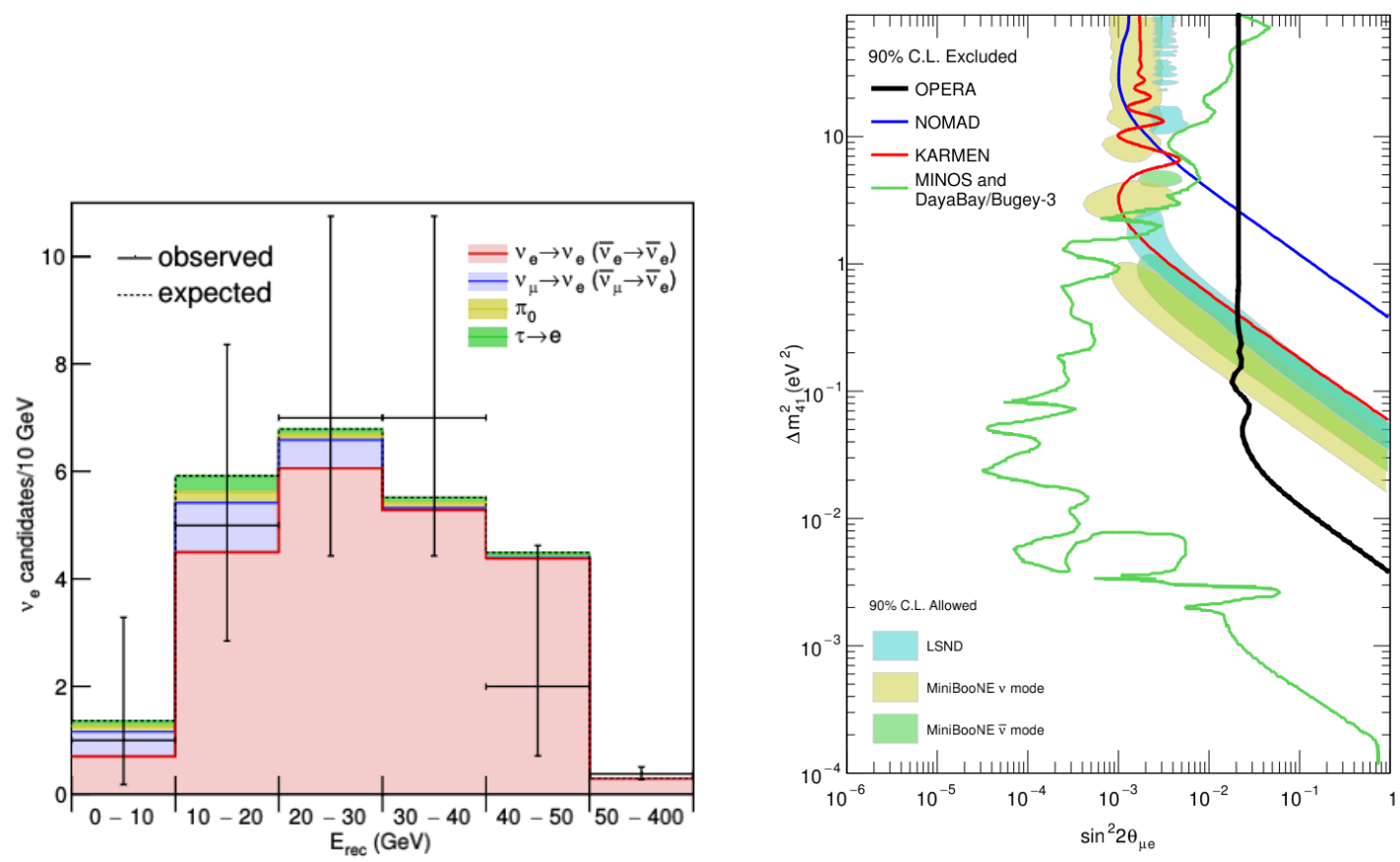

Figure 4. Left plot: reconstructed energy distribution for the observed $v_{e}$ Charged Current (CC) events. The MonteCarlo expectations for signal and background are also shown. Right plot: 90\% excluded region for the $3+1$ model by OPERA results on $v_{\mu} \rightarrow v_{e}$ oscillations. Exclusion regions obtained by NOMAD, KARMEN as well as the MINOS and DayaBay/Bugey-3 joint analysis are also shown, together with the allowed regions obtained by LSND and MiniBooNE.

Both the $v_{e}$ and the $v_{\tau}$ appearance analyses, in the $3+1$ model, were performed by means of GLoBES simulator [12,13], which also takes into account the matter effect in neutrino propagation. A likelihood is constructed using the correct parametrization of the mixing matrix (with six angles and three phases) and its maximization in the $\Delta m_{41}^{2}$ vs. $\sin ^{2}\left(2 \theta_{\mu e}\right)$ plane is performed over the full parameter space of mixing angles and phases. $\Delta m_{21}^{2}$ is fixed at its PDG value, while for $\Delta m_{31}^{2}$ a Gaussian distribution is used, also derived from the PDG [1]. Inverted and normal hierarchies of the three active neutrino masses are treated separately.

\subsection{Charged Hadron Multiplicities in Charged-Current Neutrino-Lead Interactions}

The multiplicity distribution of charged hadrons is characteristic of hadronic final states in hard scattering interactions. OPERA results can be helpful in improving models of particle production used in MonteCarlo generators. The analysis was based on a sub-sample of about 800 events with a muon in the final state identified by the muon spectrometers. A cut on $W^{2}$, the square of the invariant 
mass of hadronic system, required to be greater than $1 \mathrm{GeV}^{2} / \mathrm{c}^{4}$, was then performed in order to reject quasi-elastic interactions. Fragments from the target nuclei and recoil nuclei are excluded from the hadron shower multiplicity calculation thanks to their high ionizing power measured with emulsions. OPERA results were published in Reference [14]. The dependence of the average multiplicity $\left\langle n_{c h}\right\rangle$ on $\ln W^{2}$, shown in Figure 5-left, is approximately linear. The same is true for the dependence of the dispersion $D_{c h}=\sqrt{\left\langle n_{c h}^{2}\right\rangle-\left\langle n_{c h}\right\rangle^{2}}$ on $\left\langle n_{c h}\right\rangle$, as known from empirical observations; the plot of the dispersion is shown in Figure 5-right.
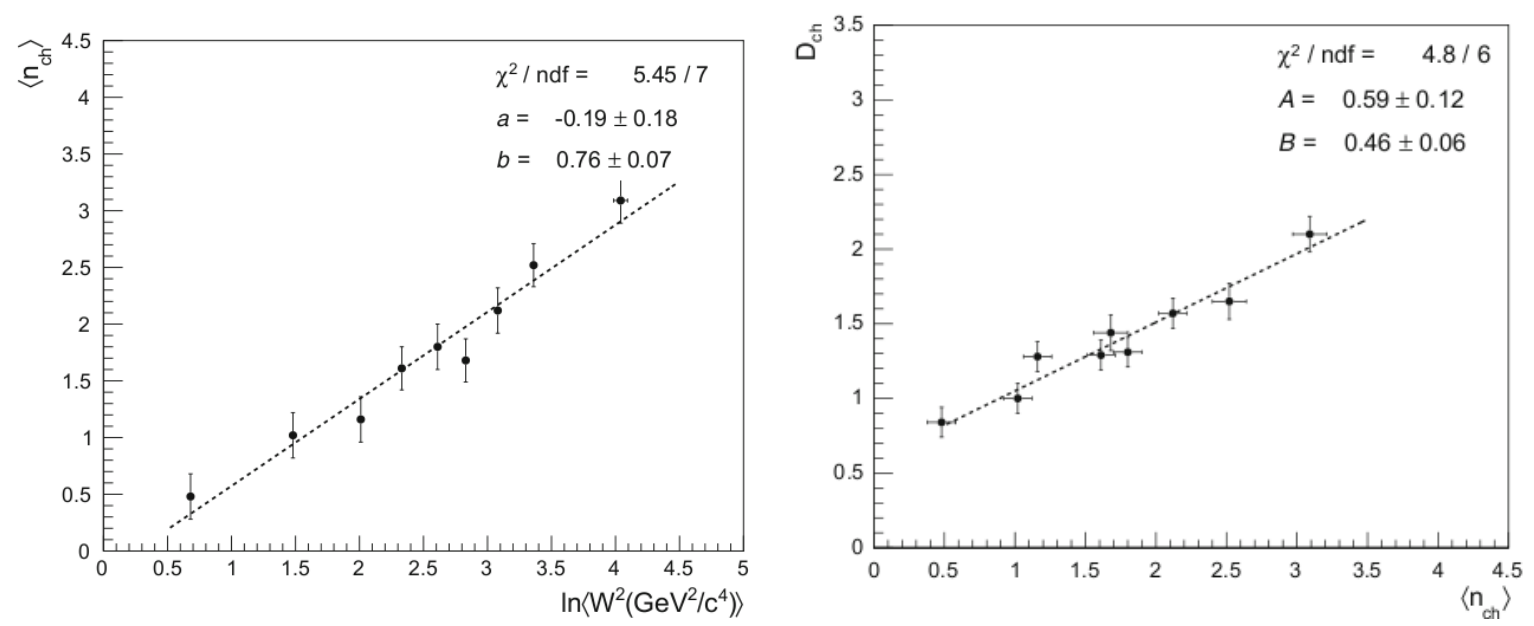

Figure 5. Left plot: average hadron multiplicity as a function of $\ln W^{2}$. The linear fit $\left\langle n_{c h}>=\right.$ $a+b \times \ln W^{2}$ is over-imposed. Right plot: dispersion as a function of the average hadron multiplicity. The linear fit $D_{c h}=A+B \times<n_{c h}>$ is over-imposed. See text for definitions.

The Koba-Nielsen-Olesen (KNO) [15] scaling, with the low energy corrections suggested in Reference [16], is also verified, i.e., the multiplicity distribution is independent of the primary energy, as reported in Figure 6, showing our results.

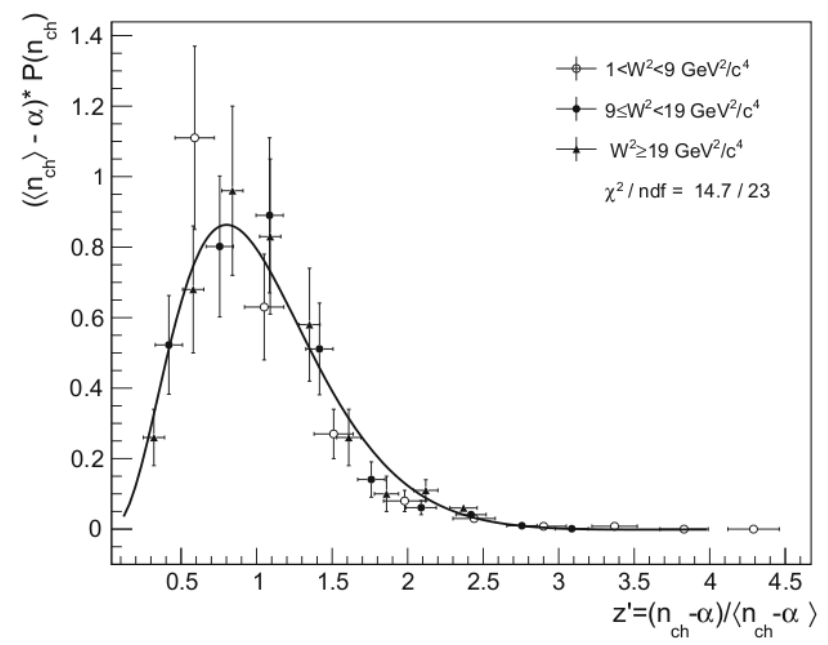

Figure 6. Koba-Nielsen-Olesen (KNO) scaling distribution. The parameter $\alpha=-A$ / $B$ is derived from the results of the fit on $D_{c h}$.

\subsection{Cosmic Ray Physics}

OPERA electronic detectors, i.e., the target trackers and the muon spectrometers, were used to detect muons produced by interactions of primary cosmic rays in the upper atmosphere. The overburden of the Gran Sasso underground laboratory, $3800 \mathrm{~m}$ water equivalent, selects muons of $\mathrm{TeV}$ energy, higher than other underground experimental sites. In addition, among all the Gran 
Sasso experiments, OPERA was the only one endowed with precision muon spectrometers, allowing the charge measurement. Using single cosmic muon events, we have measured both the charge ratio $\left(\mathrm{R}=N_{\mu^{+}} / N_{\mu^{-}}\right)$[17] and the annual modulation of the flux [18]. In Figure 7, the measured charge ratio is shown, together with the values measured by other experiments with different overburdens.

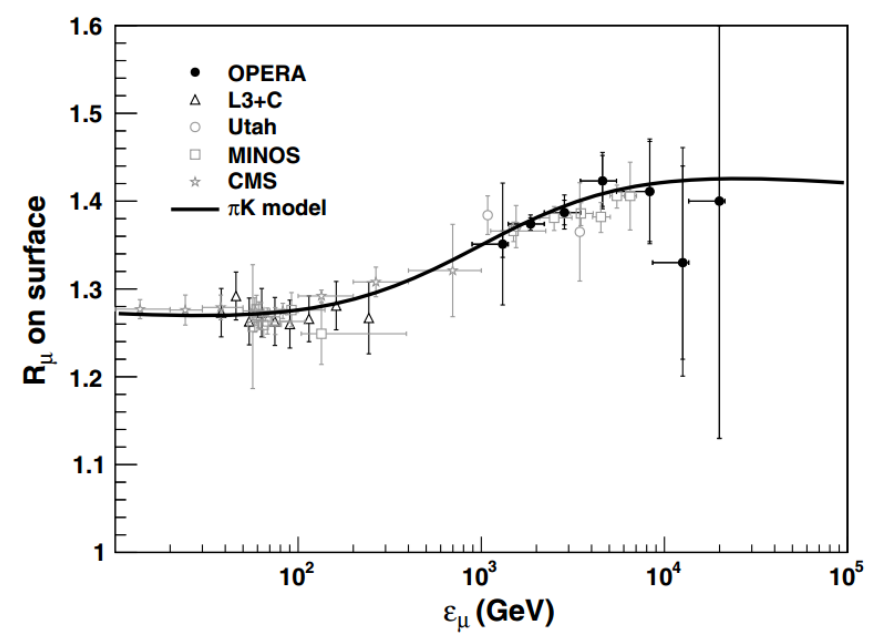

Figure 7. Muon charge ratio measured by OPERA and by other experiments as a function of the vertical surface energy $\epsilon_{\mu}$.

The flux of single cosmic ray muons in underground laboratories shows an annual modulation as a consequence of the atmospheric temperature variation, which changing the air density, modifies the proportion between $\pi / K$ mesons decays (into muons) and interactions. The amplitude of the muon flux modulation amounts to $(1.55 \pm 0.08) \%$ of the average value. The relative variations of the muon flux and of the effective atmospheric temperature (defined as the temperature averaged over the altitude according to the muon production rate) are correlated with a constant $\alpha_{T}$, depending on the average energy of the muons reaching the underground detectors. With the muons reconstructed by the OPERA electronic detectors, a value $\alpha_{T}=(0.95 \pm 0.04)$ has been measured for $1.1 \mathrm{TeV}$ muons, consistent with all the other LNGS experiments.

\section{Discussion}

The OPERA experiment was designed to observe $v_{\mu} \rightarrow v_{\tau}$ oscillations through $\tau$ appearance in a pure $v_{\mu}$ beam. The use of the nuclear emulsion technique allowed the reconstruction and the identification on an event-by-event basis of the CC interactions of all three neutrino flavors. OPERA accomplished its main task at $6.1 \sigma$ significance on $\tau$ appearance, to be compared with $4.6 \sigma$ obtained by Super-Kamiokande with a statistical analysis of atmospheric neutrinos [19].

The high average CNGS beam energy, $17 \mathrm{GeV}$, optimized for $\tau$ production and identification, is does not match the oscillation maximum at the baseline of $730 \mathrm{~km}$, and therefore OPERA results are not competitive with the precise measurements of $\theta_{23}, \Delta m_{32}^{2}$ and $\theta_{13}$ performed in disappearance mode by Super-Kamiokande [20], MINOS [21], T2K [22], NOvA [23], Daya Bay [24], RENO [25], and Double Chooz [26]. Nonetheless the higher neutrino energy can be useful to further constrain theories beyond the Standard Model. OPERA analysis in the $3+1$ neutrino model has already been published for $v_{\mu} \rightarrow v_{e}$ oscillations, excluding a large part of the region allowed by the recent MiniBoone results published in Reference [27]. It is worth noticing, however, that a more stringent exclusion limit has been obtained by MINOS and Daya Bay/Bugey-3 combining the analyses of their disappearance results on a $v_{\mu}$ beam and on reactors anti- $v_{e}$ fluxes, respectively [28]. A similar analysis for $v_{\mu} \rightarrow v_{\tau}$ is in progress.

Using the precise measurements on neutrino oscillations performed by the experiments quoted above, the estimation of $v_{\tau}$ flux impinging on the OPERA target, given by the product between the 
$v_{\mu}$ energy spectrum and the oscillation probability, has been used to measure the $v_{\tau}$ CC cross section on lead, exploiting the ten observed events. Given the low statistics, the cross section reported in Section 3.1 is averaged over the expected $v_{\tau}$ flux. The OPERA result, consistent with the Standard Model prediction as provided by GENIE neutrino MonteCarlo generator [9], can be compared to the measurement by DONuT Collaboration [29] in a beam dump experiment at FermiLab, based on the observation of $9 v_{\tau} \mathrm{CC}$ events. The DONuT experiment was using experimental techniques similar to the OPERA ones, with lead sheets as targets, alternated with nuclear emulsions. The anti-neutrino contamination was however much higher than OPERA, whose $v_{\tau}$ sample is composed of neutrinos with an expected anti-neutrino contamination of $2 \%$ in terms of interactions with the lead target. A better measurement will eventually be performed in the future by the proposed SHiP experiment [30], based on samples of more than one thousand $v_{\tau}$ and few hundreds anti- $v_{\tau} \mathrm{CC}$ interactions. The experiment is designed to separate neutrinos from anti-neutrinos by measuring the $\tau$ charge. The high amount of statistics will, for the first time, allow for the measurement of the Deep Inelastic Scattering (DIS) structure functions.

Funding: Funding is gratefully acknowledged from national agencies and Institutions supporting us, namely: Fonds de la Recherche Scientifique-FNRS and Institut Interuniversitaire des Sciences Nucleaires for Belgium; MoSES for Croatia; CNRS and IN2P3 for France; BMBF for Germany; INFN for Italy; JSPS, MEXT, the QFPU-Global COE program of Nagoya University, and Promotion and Mutual Aid Corporation for Private Schools of Japan for Japan; SNF, the University of Bern and ETH Zurich for Switzerland; the Russian Foundation for Basic Research (Grant No. 12-02-12142 ofim), the Programs of the Presidium of the Russian Academy of Sciences (Neutrino Physics and Experimental and Theoretical Researches of Fundamental Interactions), and the Ministry of Education and Science of the Russian Federation for Russia, the Basic Science Research Program through the National Research Foundation of Korea (NRF) funded by the Ministry of Science and ICT (Grant No. NRF-2018R1A2B2007757) for Korea; and TUBITAK, the Scientific and Technological Research Council of Turkey for Turkey (Grant No. 108T324).

Acknowledgments: We warmly thank CERN for the successful operation of the CNGS facility and INFN for the continuous support given by hosting the experiment in its LNGS laboratory.

Conflicts of Interest: The authors declare no conflict of interest.

\section{References}

1. Nakamura, K.; Petkov, S.T. Neutrino Masses, Mixing, and Oscillations. Available online: pdg.lbl.gov/2018/ reviews/rpp2018-rev-neutrino-mixing.pdf (accessed on 5 December 2019).

2. The Super-Kamiokande Collaboration. Evidence for Oscillation of Atmospheric Neutrinos. Phys. Lett. B 1998, 81, 1562. [CrossRef]

3. The OPERA Collaboration. The OPERA experiment in the CERN to Gran Sasso neutrino beam. J. Instrum. 2009, 4, P04018.

4. The OPERA Collaboration. Procedure for short-lived particle detection in the OPERA experiment and its application to charm decays. Eur. Phys. J. C 2014, 74, 2986. [CrossRef]

5. The OPERA Collaboration. New results on $v_{\mu} \rightarrow v_{\tau}$ appearance with the OPERA experiment in the CNGS beam. J. High Energy Phys. 2013, 2013, 036. [CrossRef]

6. The OPERA Collaboration. Discovery of $\tau$ Neutrino Appearance in the CNGS Neutrino Beam with the OPERA Experiment. Phys. Rev. Lett. 2015, 115, 121802. [CrossRef] [PubMed]

7. The OPERA Collaboration. Final Results of the OPERA Experiment on $v_{\tau}$ Appearance in the CNGS Neutrino Beam. Phys. Rev. Lett. 2018, 120, 211801. [CrossRef]

8. Longhin, A.; Paoloni, A.; Pupilli, F. Large-Angle Scattering of Multi-GeV Muons on Thin Lead Targets. IEEE Trans. Nucl. Sci. 2015, 62, 2216. [CrossRef]

9. Andreopoulos, C.; Barry, C.; Dytman, S.; Gallagher, H.; Golan, T.; Hatcher, R.; Perdue, G.; Yarba, J. The GENIE Neutrino Monte Carlo Generator: Physics and User Manual. arxiv 2015, arxiv:1510.05494.

10. The OPERA Collaboration. Limits on muon-neutrino to tau-neutrino oscillations induced by a sterile neutrino state obtained by OPERA at the CNGS beam. J. High Energy Phys. 2015, 2015, 069. [CrossRef]

11. The OPERA Collaboration. Final results of the search for $v_{\mu} \rightarrow v_{e}$ oscillations with the OPERA detector in the CNGS beam. J. High Energy Phys. 2018, 2018, 151. [CrossRef] 
12. Huber, P.; Lindner, M.; Winter, W. Simulation of long-baseline neutrino oscillation experiments with GLoBES (General Long Baseline Experiment Simulator). Comput. Phys. Commun. 2005, 167, 195. [CrossRef]

13. Huber, P.; Kopp, J.; Lindner, M.; Rolinec, M.; Winter, W. New features in the simulation of neutrino oscillation experiments with GLoBES 3.0: General Long Baseline Experiment Simulator. Comput. Phys. Commun. 2007, 177, 432. [CrossRef]

14. The OPERA Collaboration. Study of charged hadron multiplicities in charged-current neutrino-lead interactions in the OPERA detector. Eur. Phys. J. C 2018, 78, 62. [CrossRef]

15. Koba, F.; Nielsen, H.B.; Olesen, P. Scaling of multiplicity distributions in high-energy hadron collisions. Nucl. Phys. B 1972, 40, 317-334. [CrossRef]

16. Buras, A.J.; Dias de Deus, J.; Moller, R. Multiplicity scaling at low energies, a generalized Wroblewski-formula and the leading particle effect. Phys. Lett. B 1973, 47, 251-254. [CrossRef]

17. The OPERA Collaboration. Measurement of the TeV atmospheric muon charge ratio with the complete OPERA data set. Eur. Phys. J. C 2014, 74, 2933. [CrossRef]

18. The OPERA Collaboration. Measurement of the cosmic ray muon flux seasonal variation with the OPERA detector. arxiv 2018, arxiv:1810.10783.

19. The Super-Kamiokande Collaboration. A Measurement of the Tau Neutrino Cross Section in Atmospheric Neutrino Oscillations with Super-Kamiokande. Phys. Rev. D 2018, 98, 052006. [CrossRef]

20. Moriyama, S. et al. [Super-Kamiokande Collaboration]. New atmospheric and solar results from Super-Kamiokande. J. Phys. Conf. Ser. 2017, 888, 012005. [CrossRef]

21. The Minos Collaboration. Combined analysis of muon-neutrino disappearance and muon-neutrino to electron-neutrino appearance in MINOS using accelerator and atmospheric neutrinos. Phys. Rev. D 2014, 112, 191811.

22. The T2K Collaboration. Measurement of neutrino and antineutrino oscillations by the T2K experiment including a new additional sample of $v_{e}$ interactions at the far detector. Phys. Rev. D 2017, 98, 019902.

23. The NOvA Collaboration. New constraints on oscillation parameters from $v_{e}$ appearance and $v_{\mu}$ disappearance in the NOvA experiment. Phys. Rev. D 2018, 98, 032012. [CrossRef]

24. The Daya Bay Collaboration. Measurement of electron antineutrino oscillation with 1958 days of operation at Daya Bay. arxiv 2018, arxiv:1809.02261.

25. The RENO Collaboration. Measurement of neutrino mixing angle $\theta_{13}$ and mass difference $\Delta m_{e e}^{2}$ from reactor antineutrino disappearance in the RENO experiment. Nucl. Phys. B 2016, 908, 94-115. [CrossRef]

26. The Double Chooz Collaboration. Measurement of $\theta_{13}$ in Double Chooz using neutron captures on hydrogen with novel background rejection techniques. J. High Energy Phys. 2016, 2016, 163. [CrossRef]

27. The MiniBooNE collaboration. Observation of a Significant Excess of Electron-Like Events in the MiniBooNE Short-Baseline Neutrino Experiment. arxiv 2018, arxiv:1805.12028.

28. The Minos and the Daya Bay Collaborations. Limits on Active to Sterile Neutrino Oscillations from Disappearance Searches in the MINOS, Daya Bay, and Bugey-3 Experiments. Phys. Rev. Lett. 2016, 117, 151801. [CrossRef] [PubMed]

29. The DONuT Collaboration. Final tau-neutrino results from the DONuT experiment. Phys. Rev. D 2008, 78, 052002. [CrossRef]

30. The SHiP Collaboration. A facility to Search for Hidden Particles (SHiP) at the CERN SPS. arxiv 2015, arxiv:1504.04956.

(C) 2018 by the author. Licensee MDPI, Basel, Switzerland. This article is an open access article distributed under the terms and conditions of the Creative Commons Attribution (CC BY) license (http://creativecommons.org/licenses/by/4.0/). 